\title{
- Retrieval and characterization of carbon monoxide (CO) \\ vertical profiles in the Martian atmosphere from observations of PFS/MEX
}

\author{
Jimmy Bouche ${ }^{\mathrm{a}}$, Sophie Bauduin ${ }^{\mathrm{a}}$, Marco Giuranna ${ }^{\mathrm{b}}$, Séverine Robert ${ }^{\mathrm{c}}$, \\ Shohei Aoki ${ }^{c}$, Ann Carine Vandaelec ${ }^{c}$ Justin T. Erwin ${ }^{c}$, Frank Daerden ${ }^{c}$, \\ Paulina Wolkenberg ${ }^{\mathrm{b}, \mathrm{d}}$, Pierre-François Coheur ${ }^{\mathrm{a}}$ \\ ${ }^{a}$ Université libre de Bruxelles (ULB), Atmospheric spectroscopy, Service de Chimie \\ Quantique et Photophysique CP 160/09, Avenue F.D. Roosevelt 50, Bruxelles, Belgium. \\ ${ }^{b}$ National Institute of Astrophysics INAF-IAPS, Rome, Italy. \\ ${ }^{c}$ Royal Belgian Institute for Space Aeronomy, Brussels, Belgium. \\ ${ }^{d}$ Space Research Centre of Polish Academy of Sciences, Warsaw, Poland.
}

\begin{abstract}
The knowledge of the carbon monoxide (CO) abundance on Mars is essential in order to assess the processes driving the carbon cycle on the planet. Solar occultation measurements provide vertically-resolved measurements of CO from a few kilometers to higher altitudes and can be complemented by nadir measurements to enhance the spatial coverage of observations and the monitoring of the near-surface layer. Up to now, in the shortwave, CO retrievals from nadir observations have, however, mostly been performed on mean spectra and only total column abundances have been obtained. In this work we explore the possibility of exploiting nadir measurements from the Planetary Fourier Spectrometer (PFS) in the 1-0 band of CO (centered at $2143 \mathrm{~cm}^{-1}$ ) to retrieve vertical profiles of that species on individual measurement. The retrievals are performed for a set of 16 selected PFS spectra with reasonable signal-to-noise ratios by applying the Optimal Estimation Method (OEM) with appropriate constraints, built from model simulations of the Martian atmosphere. The retrieved profiles are characterized in terms of vertical sensitivity and errors. We demonstrate, in particular, that the PFS nadir measurements carry information mostly on the CO column below $15 \mathrm{~km}$, with a maximum sensitivity to the near-surface atmosphere. These measurements allow to substantially reduce the prior uncertainty on the CO abundance in this altitude range, with an estimated total retrieval error on
\end{abstract}


the column-averaged volume mixing ratio (VMR) around $10 \%$. We show that the set of retrieved VMRs are in the range of values reported from other instruments. The retrieved VMRs also capture well the known spatial and seasonal $\mathrm{CO}$ variability, which is promising in the perspective of better exploiting the exceptional set of PFS observations on Mars.

Keywords:

PFS, Mars Express, carbon monoxide, vertical profiles, Optimal

Estimation, Martian atmosphere, thermal infrared, nadir

\section{Introduction}

The first detection of carbon monoxide (CO) in the atmosphere of Mars has been made 50 years ago by [1] using ground-based observations. Since then, it has been discovered that CO plays a major role in the carbon photochemical cycle. $\mathrm{CO}$ is indeed formed by the photolysis of carbon dioxide $\left(\mathrm{CO}_{2}\right)$ and its recombination with oxygen $(\mathrm{O})$ atoms is at the center of the so-called stability problem of $\mathrm{CO}_{2}$ in the Martian atmosphere. This reaction is spin-forbidden and extremely slow compared to the recombination of $\mathrm{O}$ atoms into $\mathrm{O}_{2}$. As a consequence, without an efficient recycling process, the total amount of $\mathrm{CO}_{2}$ would have been converted into its photolysis products, and the abundance of $\mathrm{CO}$ and $\mathrm{O}_{2}$ in the Martian atmosphere would have significantly exceeded the present amount. In reality, as proposed in the 1970's by [2] and [3], the recombination of $\mathrm{CO}$ into $\mathrm{CO}_{2}$ is catalysed by odd hydrogen $\left(\mathrm{H}, \mathrm{OH}\right.$ and $\mathrm{HO}_{2}$ and generally written $\mathrm{HO}_{x}$ ) formed by the photodissociation of water vapour $\left(\mathrm{H}_{2} \mathrm{O}\right)$. These earlier models were, however, not completely satisfactory; they used either an eddy diffusion coefficient unacceptably large for the middle atmosphere of Mars [2] or an amount of $\mathrm{H}_{2} \mathrm{O}$ significantly exceeding the known values [3]. Although more recent models have solved some of these shortcomings $[4,5,6,7,8]$, the global carbon cycle on Mars is nowadays still not completely understood. The contribution of heterogeneous chemistry $[9,10]$ in the different processes at play is part of the remaining uncertainties. The recently reported but debated detection of atmospheric methane, combined with its large apparent seasonal variability (e.g. $[11,12,13]$ and references therein) has challenged further the understanding of the carbon cycle. To improve on this understanding, significantly more accurate observations of the spatial, vertical and temporal variability of the different species involved in the carbon cycle on Mars are obviously 
needed.

This has already been partly the case for $\mathrm{CO}$, for which the knowledge of spatial and seasonal variations in the Martian atmosphere has improved thanks to different remote observations, made from ground or from space in the last 15 years. Contrary to what has been considered for a long time, it was shown that $\mathrm{CO}$ was not uniformly mixed but has strong latitudinal and seasonal variations, intimately related to the condensation-sublimation cycle of $\mathrm{CO}_{2}$ at the poles. The first evidence of the variability came from ground-based observations [14], that showed a north-to-south increase of the $\mathrm{CO}$ volume mixing ratio (VMR) has been reported during the Northern summer due to the condensation of $\mathrm{CO}_{2}$ at the south polar cap. Since then, a more complete picture of the latitudinal-seasonal changes of $\mathrm{CO}$ has been brought thanks to different instruments, such as OMEGA (Observatoire pour la Minéralogie, l'Eau, les Glaces et l'Activité) [15] and PFS (Planetary Fourier Spectrometer) [16, 17, 18] on-board Mars Express (MEX), and CRISM (Compact Reconnaissance Imaging Spectrometer for Mars) [19, 20] on-board the Mars Reconnaissance Orbiter (MRO). Note also that atmospheric CO has been measured in other spectral regions, such as in the submillimeter and the microwave [21, 22, 23, 24]. These measurements in the pure rotational lines of CO have been helpful for measuring the thermal structure of the Martian atmosphere as well as wind velocities with sufficient resolution. However, there has been no systematic retrieval of the $\mathrm{CO}$ vertical profile, especially from nadir observations. The only study of this kind attempted to exploit different spectral ranges or instruments to retrieve information on the $\mathrm{CO}$ vertical distribution [25], but these synergistic approaches are difficult to implement. This is in stark contrast to Earth, where the retrieval of vertical profile is becoming standard. For CO in particular, it has been shown for thermal infrared (TIR) sounders that, in case of high temperature contrasts between the ground and the air above it (hereafter called thermal contrast), two partial columns of $\mathrm{CO}$ can be decorrelated for the lower part of the atmosphere (surface layer and rest of the troposphere) [26]. If possible on Mars, the retrieval of CO profiles, even if weakly resolved, would provide useful constraints for the models regarding the carbon chemistry in the atmosphere close to the surface, a region generally not accessible to limb/solar occultation measurements. This work examines therefore the possibility of retrieving information on the CO vertical distribution from Martian nadir TIR observations. This is performed on a small set of single PFS nadir spectra, chosen to represent different conditions of $\mathrm{CO}$ on Mars, 
in the spectral region of the $\mathrm{CO}$ fundamental vibrational band (centered at $2143 \mathrm{~cm}^{-1}$ ). The Optimal Estimation Method (OEM) [27] is used, which allows for a detailed characterization of the retrieved CO profiles in terms of error budget and vertical sensitivity. Section 2 presents the PFS instrument and section 3 is dedicated to a short description of the radiative transfer. Section 4 introduces the OEM. The selection of PFS observations and the retrieval settings are described in section 5 and the results are discussed in section 6 , with a detailed interpretation of the error budget of the retrieved CO. The conclusions of the study are summarized in section 7 .

\section{PFS instrument}

PFS [28] is a double pendulum Fourier transform interferometer on-board the Mars Express spacecraft. It circles Mars on a quasi-polar orbit with an inclination of $87^{\circ}$ and a pericentre altitude of around $250 \mathrm{~km}$. It covers a large spectral range in the infrared $\left(250-8200 \mathrm{~cm}^{-1}\right)$ thanks to two different channels: the Long Wavelength Channel (LWC, 250-1700 $\mathrm{cm}^{-1}$ ) and the Short Wavelength Channel (SWC, 1700-8200 $\mathrm{cm}^{-1}$ ). Both channels have a sampling step of $1 \mathrm{~cm}^{-1}$ and a spectral resolution of $\sim 1.3 \mathrm{~cm}^{-1}$, when no apodization function is applied, and of $\sim 1.8 \mathrm{~cm}^{-1}$ when a Hamming function is applied to the measured interferograms (as in the case of the present work). The instrument has an instantaneous field-of-view (IFOV) of about $1.6^{\circ}$ full width at half maximum (FWHM) for the SWC and of $2.8^{\circ} \mathrm{FWHM}$ for the LWC, which correspond to a spatial resolution on ground of 7 and $12 \mathrm{~km}$ respectively, when PFS is at $250 \mathrm{~km}$ from Mars. A full description of the PFS design, specification and operation can be found in [28]. Additional details about the spectral and radiometric calibration procedure for both channels may be found in $[29,30]$.

Since the beginning of its science operation, PFS has been collecting Martian atmospheric spectra, currently covering more than 7 Martian years (MY 26 to MY 34). In this work, we have selected only several PFS nadir spectra associated to different Martian conditions, especially in terms of seasonal and latitudinal variations of $\mathrm{CO}$ (see section 5.1 for details). Among the two channels, only the SWC spectral range covers the vibrational bands of $\mathrm{CO}$, as the 1-0 and the $2-0$ centered at $2143 \mathrm{~cm}^{-1}$ and at $4235 \mathrm{~cm}^{-1}$ respectively. For this study, only the stronger 1-0 band of $\mathrm{CO}$ has been considered in apodized SWC spectra. 


\section{Radiative transfer}

The monochromatic infrared radiance at wavenumber $\tilde{\nu}$ measured by PFS at the top of the Martian atmosphere (TOA), $I^{\uparrow}(\tilde{\nu}, \theta$, TOA), can be described by the sum of two terms: 1) the transmission of the radiance emitted and reflected by the Martian surface, and 2) the contribution of scattering and emission by the atmosphere. This is expressed by the equation of the radiative transfer:

$$
\begin{aligned}
I^{\uparrow}(\tilde{\nu}, \theta, \mathrm{TOA})= & I^{\uparrow}(\tilde{\nu}, \theta, 0) t(\tilde{\nu}, \theta, 0, \mathrm{TOA}) \\
& +\int_{0}^{\mathrm{TOA}} J(\tilde{\nu}, \Omega, z) \frac{\partial t(\tilde{\nu}, \theta, z, \mathrm{TOA})}{\partial z} \mathrm{~d} z,
\end{aligned}
$$

where $\theta$ is the ground zenith angle, $t(\tilde{\nu}, \theta, 0, T O A)$ is the transmittance of the Martian atmosphere, $t(\tilde{\nu}, \theta, z$, TOA $)$ is the transmittance between altitude $z$ and TOA, $J(\tilde{\nu}, \Omega, z)$ is the atmospheric source term which depends on thermal emission and scattering and where $\Omega$ represents the geometric angles. Note that in this work, only clear or almost clear atmospheres (see section 5.1) have been analysed. In this case, scattering can be neglected and the atmospheric source function can be approximated by a Plank function $B(\tilde{\nu}, T)$. The radiance at the surface, $I^{\uparrow}(\tilde{\nu}, \theta, 0)$, takes into account the emission of the ground and the reflection of the solar incoming radiation as follows:

$$
I^{\uparrow}(\tilde{\nu}, \theta, 0)=\epsilon(\tilde{\nu}) B\left(T_{s}\right)+(1-\epsilon(\tilde{\nu})) I_{0}^{\downarrow \dagger}(\tilde{\nu})+r\left(\tilde{\nu}, \theta, \phi, \theta^{\star}, \phi^{\star}\right) F_{0}^{\downarrow \star}(\tilde{\nu}),
$$

where $B\left(T_{s}\right)$ is the Planck function at surface temperature $T_{s}, \epsilon(\tilde{\nu})$ is the wavenumber dependent emissivity of the Martian surface, $I_{0}^{\downarrow \dagger}(\tilde{\nu})$ is the mean radiance associated to the downward flux of the atmosphere and $F_{0}^{\downarrow \star}(\tilde{\nu})$ is the solar flux reaching Mars' surface. $r\left(\tilde{\nu}, \theta, \phi, \theta^{\star}, \phi^{\star}\right)$ is the effective reflectivity of the surface. $\theta^{\star}$ and $\phi^{\star}$ are respectively the zenithal and azimuthal angles of the Sun, and $\phi$ is the azimuthal angle of the spacecraft.

In order to calculate the TOA radiance, equation 1 is discretized into a set of layers, in which the averaged temperature and pressure and the partial columns of molecular species are calculated. The upwelling radiance at the top of layer $i$ is estimated as

$$
I_{i}^{\uparrow}=B\left(\bar{T}_{i}\right)+\left(I_{i-1}^{\uparrow}-B\left(\bar{T}_{i}\right)\right) \tilde{t}_{i},
$$


where $B\left(\bar{T}_{i}\right)$ is the emission of layer $i$ approximated as a blackbody at the averaged temperature $\bar{T}_{i}$. The effective transmittance $\tilde{t}_{i}$ of layer $i$ is calculated using

$$
\tilde{t}_{i}=\exp \left[-\sum_{j} P C_{i, j} \sum_{j, k} \sigma_{j, k}\left(\tilde{\nu}, \bar{P}_{i}, \bar{T}_{i}\right)\right],
$$

where $j$ is associated to the molecular species and $k$ to the relevant absorption lines at the chosen $\tilde{\nu}$. $P C_{i, j}$ is the partial column of species $j$ in layer $i$ and $\sigma_{j, k}\left(\tilde{\nu}, \bar{P}_{i}, \bar{T}_{i}\right)$ is the absorption cross section of that species $\left(\mathrm{cm}^{2} /\right.$ molecules $)$ calculated for the mean parameters of layer $i$.

In this work, the Atmosphit software has been used for radiative transfer calculations. It is a versatile line-by-line radiative transfer code initially developed for Earth atmosphere, but which can easily be adpated for Mars. Details about the Atmosphit software can be found in [31], [32] and [33]. For this work, the emissivity and the effective reflectivity introduced in equation 2 are considered constant with wavenumber, and for the latter, a combined contribution of lambertian and specular reflections of the Sun radiation is assumed.

\section{Retrieval method: Optimal Estimation}

\subsection{Background}

The method used in this work to retrieve the vertical profiles of $\mathrm{CO}$ is the OEM [27]. The idea of this method is to find the CO profile that is the most consistent with both PFS observations and a certain prior knowledge of its distribution in the Martian atmosphere. This is done by minimizing the following cost function $\phi$

$$
\phi=(\mathbf{y}-\mathrm{F}(\mathbf{x}, \mathbf{b}))^{\mathrm{T}} \mathbf{S}_{\boldsymbol{\epsilon}}{ }^{-1}(\mathbf{y}-\mathrm{F}(\mathbf{x}, \mathbf{b}))+\left(\mathbf{x}-\mathbf{x}_{\mathbf{a}}\right)^{\mathrm{T}} \mathbf{S}_{\mathbf{a}}{ }^{-1}\left(\mathbf{x}-\mathbf{x}_{\mathbf{a}}\right),
$$

where $\mathbf{y}$ is the measurement vector, consisting of the PFS spectral radiances, $\mathbf{x}$ is the atmospheric state vector, containing the variables to be retrieved, i.e. the CO profile but also the other parameters (see section 5.3), b includes all the other fixed atmospheric variables that influence PFS measurements, $\mathbf{x}_{\mathbf{a}}$ is the a priori state vector (including the a priori profile of $\mathrm{CO}$ ) and $\mathrm{F}$ is the forward radiative transfer model. The covariance matrices, $\mathbf{S}_{\boldsymbol{\epsilon}}$ and $\mathbf{S}_{\mathbf{a}}$, are respectively the measurement and the a priori covariance matrices. 
The minimization of $\phi$ is iteratively performed by the Atmosphit software using the numerical Gauss-Newton method for moderately non-linear cases [27]. At each iteration, a new state vector is evaluated using

$$
\mathbf{x}_{i+1}=\mathbf{x}_{\mathbf{a}}+\left(\mathbf{K}_{i}^{\mathrm{T}} \mathbf{S}_{\boldsymbol{\epsilon}}{ }^{-1} \mathbf{K}_{i}+\mathbf{S}_{\mathbf{a}}{ }^{-1}\right)^{-1} \mathbf{K}_{i}^{\mathrm{T}} \mathbf{S}_{\boldsymbol{\epsilon}}{ }^{-1}\left[\mathbf{y}-\mathrm{F}\left(\mathbf{x}_{i}, \mathbf{b}\right)+\mathbf{K}_{i}\left(\mathbf{x}_{i}-\mathbf{x}_{\mathbf{a}}\right)\right]
$$

where $\mathbf{K}$ is the Jacobian matrix, whose rows correspond to the derivatives of the TOA radiance with respect to the retrieved variables. The convergence is reached when the absolute difference between the forward models of the two last iterations is lower than $20 \%$ of the noise. Note that Atmosphit does not retrieve the partial column in each layer directly but instead a scaling factor for each layer, which is applied to the a priori profile at the end of each iteration.

\subsection{Characterization of the retrievals}

\subsubsection{Vertical sensitivity}

The OEM offers a very adequate framework to characterize the retrieved CO profiles in terms of vertical sensitivity. This is done in this work mostly in terms of the averaging kernel matrix A. It is defined as the sensitivity of the retrieved state (hereafter symbolized by $\hat{\mathbf{x}}$ ) to the true state $(\mathbf{x})$ and can be calculated as

$$
\mathbf{A}=\frac{\partial \hat{\mathbf{x}}}{\partial \mathbf{x}}=\left(\mathbf{K}^{\mathrm{T}} \mathbf{S}_{\epsilon}{ }^{-1} \mathbf{K}+\mathbf{S}_{\mathbf{a}}{ }^{-1}\right)^{-1} \mathbf{K}^{\mathrm{T}} \mathbf{S}_{\epsilon}{ }^{-1} \mathbf{K} .
$$

The rows of A define an averaging kernel function (AVK) for each element of $\hat{\mathbf{x}}$, which peaks at the altitude from where most of the information is coming. In other words, the AVK provide an estimation of the vertical sensitivity of the retrieval and peak at the altitude of maximum sensitivity. The trace of the A matrix gives the degree of freedom for signal (DOFS), defined as the number of independent pieces of information in the retrieved profile.

\subsubsection{Error analysis}

As for the vertical sensitivity, a complete error budget analysis can be performed with the OEM, by separating different sources of errors from the total [27]. The following sources of errors have been evaluated:

1. The smoothing error: due to the limited vertical resolution of nadir observations, the retrieved state is a smoothed estimate of the true 
state. The resulting error can be characterized by the covariance matrix $\mathbf{S}_{\mathbf{s}}$, calculated from the $\mathbf{A}$ and $\mathbf{S}_{\mathbf{a}}$ matrices as follows

$$
\mathbf{S}_{\mathbf{s}}=(\mathbf{A}-\mathbf{I}) \mathbf{S}_{\mathbf{a}}(\mathbf{A}-\mathbf{I})^{\mathrm{T}} .
$$

2. The measurement error, which directly propagates from the instrumental noise. Its associated covariance matrix can be written as:

$$
\mathbf{S}_{\mathbf{m}}=\mathrm{GS}_{\epsilon} \mathbf{G}^{\mathrm{T}},
$$

where $\mathbf{G}$ is the gain matrix, whose rows are the derivatives of the retrieved state with respect to the measurement. Note that in this work, forward modelling errors are included in the $\mathbf{S}_{\epsilon}$ matrix (see section 5.3). The covariance matrix $\mathbf{S}_{\mathbf{m}}$ therefore describes the total error from the measurement noise and the forward model.

3. The error from the fitted parameters: this type of error comes from the interferences existing between the retrieved $\mathrm{CO}$ profile and the rest of the state vector (other retrieved parameters), such as the surface temperature (see section 5.3) [34]. Its covariance matrix is estimated using the non-diagonal blocs $\left(\mathbf{A}_{\mathbf{i r}}\right)$ of the AVK matrix (see [34] for details):

$$
\mathbf{S}_{\mathrm{f}}=\mathbf{A}_{\mathrm{ir}} \mathbf{S}_{\mathrm{ra}} \mathbf{A}_{\mathrm{ir}}{ }^{\mathrm{T}},
$$

where $\mathbf{i}$ is the part of the state vector under consideration (the CO profile), $\mathbf{r}$ represents the rest of the state vector (the surface temperature and the $\mathrm{CO}_{2}$ profile, see section 5.3), and $\mathbf{S}_{\mathbf{r a}}$ is the a priori covariance matrix of $\mathbf{r}$.

The sum of the covariance matrices associated to these different uncertainties provide the total retrieval error covariance matrix:

$$
\mathbf{S}_{\mathbf{T}}=\mathbf{S}_{\mathrm{s}}+\mathbf{S}_{\mathrm{m}}+\mathbf{S}_{\mathrm{f}}
$$

The square root of the diagonal elements of $\mathbf{S}_{\mathbf{T}}$ can be seen as the error on the retrieved $\mathrm{CO}$ at each altitude.

\section{Spectra selection and retrieval set-up}

\subsection{Selection of spectra}

$\mathrm{CO}$ vertical profiles have been retrieved from a small set of single nadir PFS observations. These observations have been chosen among all PFS measurements recorded between 10 January 2004 (MY 26) and 18 June 2017 (MY 
34), following a 2 step procedure. The first step consists in finding spectra with almost clear sky conditions and sufficient signal-to-noise ratio (SNR) to detect CO lines in the 1-0 band. For this, only PFS observations associated to dust and ice column-integrated optical depths $\left(\tau_{\mathrm{d}}\right.$ and $\left.\tau_{\mathrm{i}}\right)$ lower than 0.15 have been considered. These two parameters were provided as part of the PFS level 2 (L2) products by the National Institute of Astrophysics INAFIAPS $[18,35,36]$. Then, to guarantee sufficient SNR, only spectra meeting the following three criteria were kept:

- The radiance value at $2165.7 \mathrm{~cm}^{-1}$ (corresponding to a CO line) has to be larger than 3 times the value of the noise, which was estimated to be $0.025 \mathrm{erg} \mathrm{s}^{-1} \mathrm{sr}^{-1} \mathrm{~cm}^{-2} \mathrm{~cm}$ based on the standard deviation at $2165 \mathrm{~cm}^{-1}$ of an ensemble PFS spectra. This first criterion proves useful to discard PFS spectra with low radiance, but does not inform on the strength of the CO absorption.

- The strength of the CO line at $2165.7 \mathrm{~cm}^{-1}$, calculated in the brightness temperature (BT) space, has to be larger than $2.2 \mathrm{~K}$ (the noise equivalent temperature difference is estimated to be $1.1 \mathrm{~K}$ at $280 \mathrm{~K}$ ). This strength is calculated as the difference $(\Delta \mathrm{BT})$ between the BT at $2165.7 \mathrm{~cm}^{-1}$ and the average brightness temperature of two channels chosen for the baseline $\left(2163.6 \mathrm{~cm}^{-1}\right.$ and $\left.2167.8 \mathrm{~cm}^{-1}\right)$.

- The surface temperature from the PFS L2 product has to be larger than $240 \mathrm{~K}$ to remove spectra with very low surface contribution.

A total of around 80000 spectra in the 1-0 band, corresponding to $8 \%$ of all PFS observations are passing these selection criteria. Interestingly they cover a complete seasonal cycle and all latitudes, except polar winters. Note that with these first criteria, only daytime observations are selected; night-time observations are associated with lower SNR and have been discarded. Note finally that the possibility to apply less stringent selection criteria to extend the analysis on additional PFS observations will be tested in future studies.

After this first step, the selected PFS observations have been sorted in 5 latitude bands for the 4 seasons, as: the North Pole $\left(60^{\circ}\right.$ to $\left.90^{\circ}\right)$, the mid-latitudes of the Northern hemisphere $\left(30^{\circ}\right.$ to $\left.60^{\circ}\right)$, the Equator $\left(-30^{\circ}\right.$ to $\left.30^{\circ}\right)$, the mid-latitudes of the Southern hemisphere $\left(-60^{\circ}\right.$ to $\left.-30^{\circ}\right)$ and the South Pole $\left(-90^{\circ}\right.$ to $\left.-60^{\circ}\right)$. The seasons are defined according to the solar longitude $\left(\mathrm{L}_{s}\right)$ : Northern spring/Southern Autumn $\left(0^{\circ}-90^{\circ}\right)$, Northern 
summer/Southern winter $\left(90^{\circ}-180^{\circ}\right)$, Northern autumn/Southern spring $\left(180^{\circ}-270^{\circ}\right)$ and Northern winter/Southern summer $\left(270^{\circ}-360^{\circ}\right)$. For each latitude band and each season, only one spectrum has been selected to restrict the number of cases. Note that there were no spectra passing the criteria for the autumn and winter at both poles, characterized by very low source signals, especially during the polar night. The 16 selected spectra for this prospective study are listed in Table 1 and shown in Figure 1. Most of them present a similar radiance of around 0.6-1.0 $\mathrm{erg} \mathrm{s}^{-1} \mathrm{sr}^{-1} \mathrm{~cm}^{-2} \mathrm{~cm}$ in the CO band; the three with the larger radiances (yellow curves except for the mid-latitudes of the Northern hemisphere) are associated to scenes with high surface temperature. All 16 spectra show very clear CO lines as expected and have corresponding dust and ice integrated opacities significantly smaller than 0.15 . The CO 1-0 band consists of the $\mathrm{P}$ and $\mathrm{R}$ branches, which are both well seen in the PFS spectra, respectively in the ranges $2020-2139 \mathrm{~cm}^{-1}$ and $2147-2240 \mathrm{~cm}^{-1}$ (see Figure 1a). The spectral range on the longwave side of the $\mathrm{CO}_{2} \nu_{3}$ band (before $2200 \mathrm{~cm}^{-1}$ ) contains some other $\mathrm{CO}_{2}$ absorption features; the strongest are labelled in Figure 1a.

Table 1: Selected PFS spectra. $I_{2165}$ stands for the value of the radiance at $2165.7 \mathrm{~cm}^{-1}$. $\triangle \mathrm{BT}$ is the brightness temperature difference between a $\mathrm{CO}$ line at $2165.7 \mathrm{~cm}^{-1}$ and two baseline channels (see text for details); $\tau_{\mathrm{d}}$ and $\tau_{\mathrm{i}}$ are the dust and ice integrated opacities $[35,18,36] ; \mathrm{L}_{s}$ is the solar longitude, $\mathrm{T}_{\mathrm{s}}$ is the surface temperature $[18,35,36], \mathrm{MY}$ is the Martian year, LT is the local time and the longitudes are East.

\begin{tabular}{|c|c|c|c|c|c|c|c|c|c|c|}
\hline Lat. band & $\begin{array}{l}\mathbf{L}_{s} \\
\left({ }^{\circ}\right)\end{array}$ & $\begin{array}{l}\text { Latitude } \\
\quad\left({ }^{\circ}\right)\end{array}$ & $\begin{array}{l}\text { Longitude } \\
\left({ }^{\circ}\right)\end{array}$ & MY & $\begin{array}{l}\text { LT } \\
\text { (h) }\end{array}$ & $\begin{array}{l}\mathbf{T}_{\mathrm{s}} \\
(\mathrm{K})\end{array}$ & $\begin{array}{l}\Delta \mathbf{B T} \\
(\mathbf{K})\end{array}$ & $\begin{array}{c}I_{2165} \\
\left(\mathrm{erg} \mathrm{s}^{-1} \mathrm{sr}^{-1} \mathrm{~cm}^{-2} \mathrm{~cm}\right)\end{array}$ & $\tau_{\mathrm{d}}$ & $\tau_{\mathrm{i}}$ \\
\hline \multirow{2}{*}{ N Pole $\left(60^{\circ}-90^{\circ}\right)$} & 68.07 & 63.56 & 53.35 & 32 & 15.44 & 251 & 3.00 & 0.548 & 0.091 & 0.052 \\
\hline & 104.70 & 72.81 & 53.45 & 32 & 11.85 & 245 & 3.22 & 0.503 & 0.055 & 0.026 \\
\hline \multirow{4}{*}{$\mathrm{N}$ mid lat. $\left(30^{\circ}-60^{\circ}\right)$} & 59.66 & 52.81 & -40.13 & 32 & 15.97 & 254 & 3.69 & 0.612 & 0.067 & 0.013 \\
\hline & 135.72 & 42.09 & 31.16 & 30 & 15.77 & 257 & 3.29 & 0.606 & 0.003 & 0.063 \\
\hline & 181.22 & 31.62 & 8.12 & 31 & 9.26 & 252 & 4.43 & 0.750 & 0.067 & 0.024 \\
\hline & 330.24 & 30.09 & -86.76 & 31 & 10.02 & 245 & 3.16 & 0.561 & 0.033 & 0.054 \\
\hline \multirow{4}{*}{ Equator $\left(-30^{\circ}-30^{\circ}\right)$} & 65.89 & -6.72 & -5.32 & 32 & 15.25 & 256 & 3.61 & 0.642 & 0.055 & 0.096 \\
\hline & 132.65 & -1.92 & 58.51 & 28 & 10.16 & 259 & 3.29 & 0.790 & 0.043 & 0.021 \\
\hline & 209.13 & -5.69 & 132.43 & 30 & 10.34 & 271 & 3.35 & 1.408 & 0.066 & 0.014 \\
\hline & 324.27 & 2.61 & 92.32 & 31 & 10.55 & 253 & 3.50 & 0.784 & 0.044 & 0.011 \\
\hline \multirow{4}{*}{$\mathrm{S}$ mid lat. $\left(-60^{\circ}--30^{\circ}\right)$} & 2.12 & -40.05 & -119.41 & 30 & 14.19 & 256 & 3.48 & 0.749 & 0.023 & 0.056 \\
\hline & 155.82 & -35.69 & -155.81 & 31 & 11.02 & 248 & 2.32 & 0.530 & 0.026 & 0.064 \\
\hline & 212.85 & -47.88 & 151.47 & 30 & 9.98 & 264 & 2.54 & 0.934 & 0.125 & 0.006 \\
\hline & 334.42 & -45.38 & 118.75 & 31 & 10.26 & 253 & 3.60 & 0.766 & 0.081 & 0.049 \\
\hline \multirow{2}{*}{ S Pole $\left(-90^{\circ}--60^{\circ}\right)$} & 262.90 & -62.63 & -134.47 & 31 & 15.86 & 275 & 2.36 & 1.403 & 0.129 & 0.009 \\
\hline & 305.11 & -76.37 & 26.78 & 32 & 10.41 & 252 & 3.77 & 0.832 & 0.034 & 0.019 \\
\hline
\end{tabular}




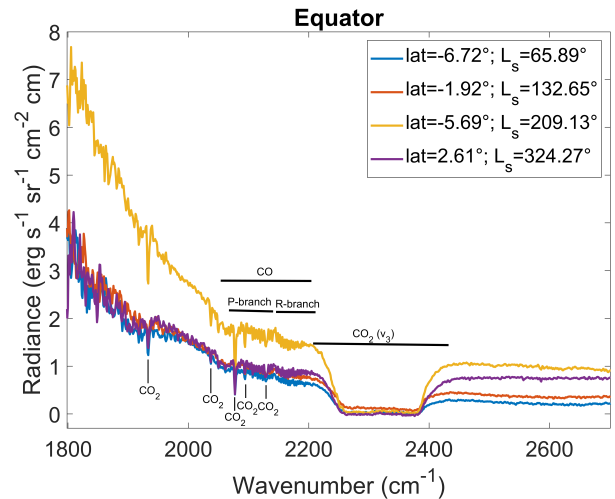

(a)

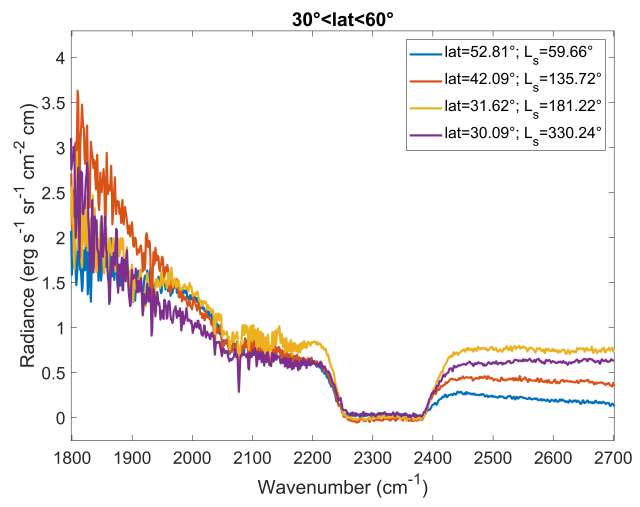

(c)

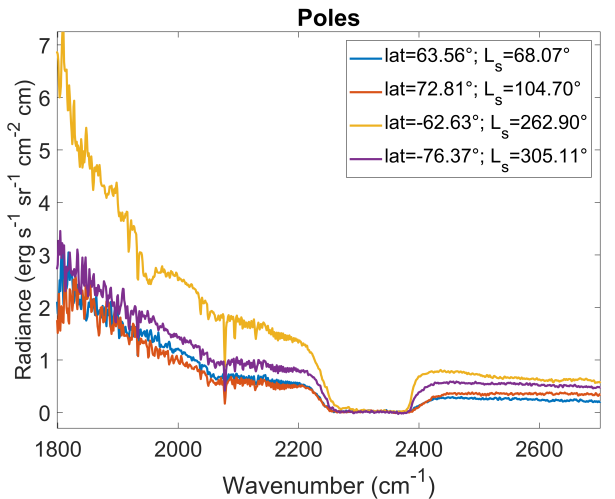

(b)

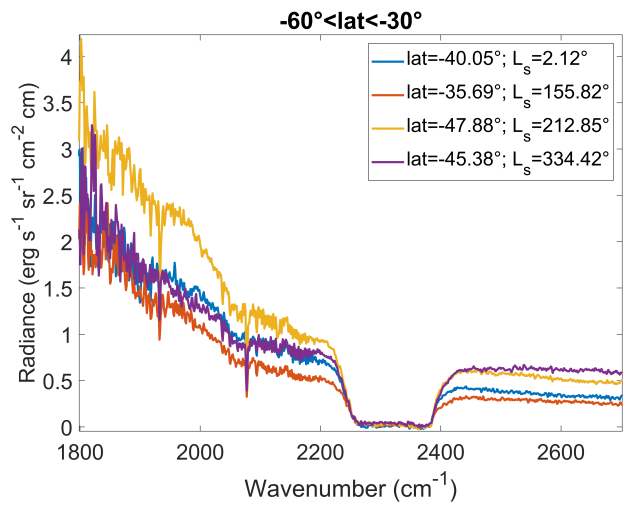

(d)

Figure 1: Selected spectra for each band of latitude; see also Table 1. Panel (b) shows the spectra selected for both North and South poles.

\subsection{Input to the radiative transfer}

The radiative transfer equation is solved for an atmosphere extending from $0 \mathrm{~km}$ to $60 \mathrm{~km}$. For each single PFS spectrum, the temperature profile is fixed to the one retrieved independently by INAF-IAPS from the corresponding spectrum recorded by the LWC (see $[35,36,37]$ for details). In the spectral range of the 1-0 band of $\mathrm{CO}$, there are lines of the $\nu_{2}$ vibrational band of water vapour $\left(\mathrm{H}_{2} \mathrm{O}\right.$; band centered at $\left.1595 \mathrm{~cm}^{-1}\right)$ and $\nu_{1}+\nu_{3} \mathrm{com}-$ bination band of ozone $\left(\mathrm{O}_{3}\right.$; centered at $\left.2105 \mathrm{~cm}^{-1}\right)$ that exist. Although their contribution is close from being negligible, without observable spectral features in the spectral range, it has been taken into account in the radiative 
transfer calculations by fixing the concentrations of the two species to climatological values. The most adequate value of surface emissivity has also to be considered, as errors on this parameter will propagate on the retrieved values of surface temperature and effective reflectivity, and hence ultimately on the retrieved CO vertical profile. The Planetary SUrface Portal [38, 39] provides global maps of the emissivity of the surface at $2000 \mathrm{~cm}^{-1}$ on spatial grids of $0.025^{\circ} \times 0.025^{\circ}$ resolution; they were built from OMEGA/MEX observations taken over 3 Martian years (MY 27-30) [40]. The emissivity values for the grid box corresponding to the selected PFS spectra have been considered in the retrieval. The spectroscopic parameters have been taken from HITRAN 2016 [41], including the pressure broadening due to $\mathrm{CO}_{2}$ [42].

The PFS spectra analysed in this work are apodized by an Hamming function. We have tested two different formulations of the ILS, which ultimately were shown to give very similar quality of fits. An example of the residuals (observed-calculated) obtained after fitting is shown in Figure 2. The first corresponds to the ILS formulation provided by INAF-IAPS [43] and which is the recommended one for the community; it is similar to the expected theoretical apodized ILS for PFS, except slightly asymmetric, and leads to the best fit for half of the 16 spectra. The second one (hereafter called "HG") stems from the theoretical Hamming function, but slightly modified with an off-axis angle (misalignment in the interferometer), to introduce a small asymmetry in the function [44], and with second-order auto-apodization effects. This second one leads to the best fits for the other half of the 16 spectra analysed in this work. For consistency with previous works, all results presented in the following are those obtained using the ILS provided by INAF-IAPS. The comparison between the two sets of fits is used later in section 6.2.3 to discuss the error possibly introduced by an imperfect ILS. 


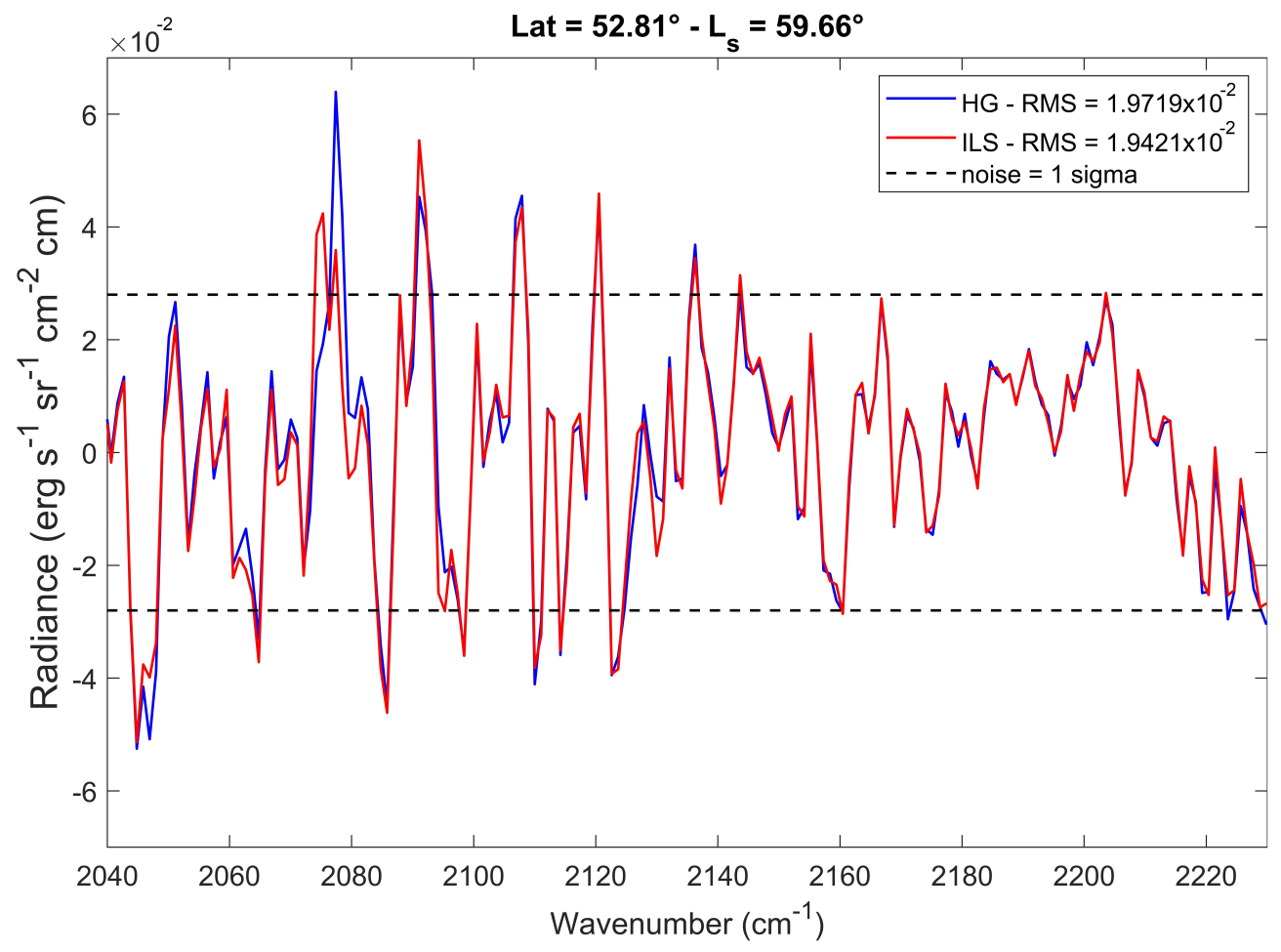

Figure 2: Example of residuals between the observed and fitted spectra calculated for the case using the HG function (blue) and the ILS provided by the INAF-IAPS (red). The RMS are shown for each case.

\subsection{Retrievals settings}

The retrieval of CO vertical profiles has been performed in two steps. In the first step, a large spectral range from 2040 to $2600 \mathrm{~cm}^{-1}$ was adjusted with the objective to obtain an accurate value for the effective surface reflectivity, which can then be used in the second step. The a priori value of the reflectivity has been set to 0.023 and its associated variability to $20 \%$. The retrieved reflectivity is fed into the second and main step, which consists in the retrieval of the vertical profile of CO. It is performed on a shorter spectral range, extending from 2040 to $2230 \mathrm{~cm}^{-1}$. Specifically, the CO vertical profiles are retrieved for $2 \mathrm{~km}$ thick layers from 0 to $24 \mathrm{~km}$; the rest of the profile is kept fixed. The a priori profile $\mathbf{x}_{\mathbf{a}}$ and covariance matrix $\mathbf{S}_{\mathbf{a}}$ used to constrain the $\mathrm{CO}$ retrieval have been built from a global ensemble of modelled profiles from the Mars Climate Database (MCD, version 5.3) [7, 45] for one Martian year (climatology solar average scenario). These profiles were 
simulated on a spatial grid of $3.75^{\circ} \times 5.625^{\circ}$ (latitude $\times$ longitude) for one day per Martian month (defined as a period of $30^{\circ} \mathrm{L}_{s}$ ). This day corresponds to the centered $\mathrm{L}_{s}$ of the month and CO profiles were calculated every 2 Martian hours (1 Martian hour is defined as $1 / 24^{\text {th }}$ of a sol), where daytime and nighttime are included. This results in an a priori covariance matrix with large but representative variability, that can be applied without changes for all observations on Mars. In total, 451584 CO profiles have been considered. The resulting $\mathbf{x}_{\mathbf{a}}$ profile and the $\mathbf{S}_{\mathbf{a}}$ matrix ${ }^{1}$ are shown in Figure 3 for the $2 \mathrm{~km}$ thick layers that have been retrieved over the altitude range from 0 to $24 \mathrm{~km}$. The a priori profile shows an almost uniform vertical distribution of $\mathrm{CO}$ at a volume mixing ratio (VMR) of about $700 \mathrm{ppm}$ between 5 and $15 \mathrm{~km}$. Close to the surface, the VMR increases to about $750 \mathrm{ppm}$ and from $15 \mathrm{~km}$ of height, it starts to increase slightly due to the photodissociation of $\mathrm{CO}_{2}$ at higher altitudes. The largest a priori variability is at the surface, where it reaches $57 \%$. It then decreases with altitude and drops to a minimum of $20 \%$ in the $12-14 \mathrm{~km}$ layer. From this minimum, the a priori variability starts to increase again. Also noteworthy and of high relevance for this work is that the $\mathbf{S}_{\mathbf{a}}$ presents non-negligible correlations between the different layers, meaning that the value of the profile retrieved at one given altitude will be influenced significantly from layers above and below. On a minor point, note that the $\mathbf{S}_{\mathbf{a}}$ originally built from the model had unphysical correlation between the surface layer and the very high atmosphere, which were removed.

In the main step of the retrieval, in addition to the $\mathrm{CO}$ profile, the surface temperature and the profile of $\mathrm{CO}_{2}$ have been retrieved simultaneously, which allows recalculating more accurately the CO VMR at the last iteration. The a priori uncertainty on the surface temperature has been set to $20 \%$. The a priori vertical profile of $\mathrm{CO}_{2}$ and its associated variability have been determined using the model output, in the same way as for CO (see above). The $\mathrm{CO}_{2}$ a priori profile is almost uniform, with a mole fraction of around 0.963 , and the prior uncertainty is very small, largest close to the surface $(2.2 \%)$ and then around $0.5 \%$ at higher altitudes. Despite the low variability allowed on the $\mathrm{CO}_{2}$ profile, fitting the latter slightly improved the quality of the fits in most cases. Finally, the $\mathbf{S}_{\boldsymbol{\epsilon}}$ has been considered diagonal

\footnotetext{
${ }^{1}$ The $\mathbf{S}_{\mathbf{a}}$ is expressed in multiplicative factor. $\sqrt{{\sigma_{\mathbf{a}}}^{2}} \times 100 \%$ represents the variability on the a priori profile.
} 
with a value for $\boldsymbol{\sigma}_{\boldsymbol{\epsilon}}$ of $0.028 \mathrm{erg} \mathrm{s}^{-1} \mathrm{sr}^{-1} \mathrm{~cm}^{-2} \mathrm{~cm}$, larger than the noise value mentioned in section 5.1 to take into account forward modelling errors.
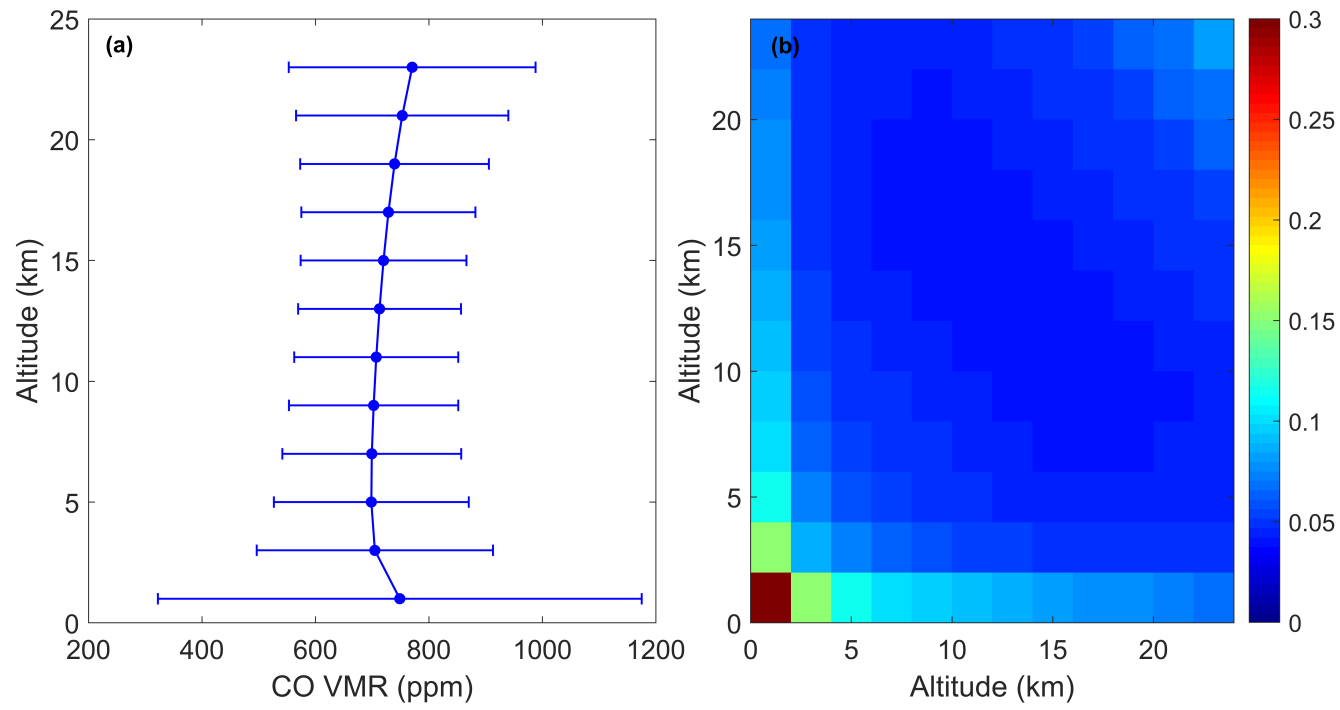

Figure 3: A priori profile (left) and covariance matrix (right) built using a representative ensemble of $\mathrm{CO}$ profiles from the MCD database (climatology solar average scenario). The matrix is expressed in multiplicative factor. The error bars on the profile correspond to the square root of the diagonal elements of the matrix, applied on the VMR.

\section{Results}

\subsection{Spectral fittings and retrieved profiles}

With this choice of retrieval parameters, the 16 spectra are fitted well, with the RMS of the residual varying between $0.019 \mathrm{erg} \mathrm{s}^{-1} \mathrm{sr}^{-1} \mathrm{~cm}^{-2} \mathrm{~cm}$ and $0.046 \mathrm{erg} \mathrm{s}^{-1} \mathrm{sr}^{-1} \mathrm{~cm}^{-2} \mathrm{~cm}$. Among all spectra, 9 have a RMS lower than the level of $\boldsymbol{\sigma}_{\boldsymbol{\epsilon}}$ considered. Figure 4 illustrates the quality of the fits for the two extreme cases, with respectively the lowest (Figure 4.a) and largest RMS (Figure 4.b) obtained. For the lowest RMS case, the residual of the fit lies well within the level of $\pm \boldsymbol{\sigma}_{\boldsymbol{\epsilon}}$ we have considered. For the highest RMS case, some spectral features remain above the noise level. This is the case in the $\mathrm{R}$ branch of the $\mathrm{CO}$ band, where the fitted spectrum is too high in radiance, mainly due to the difficulty of reproducing accurately the baseline of the spectrum, which is affected by the surface emissivity, reflectivity and temperature but also the $\mathrm{CO}_{2}$ continuum. It is worth noting that the surface 


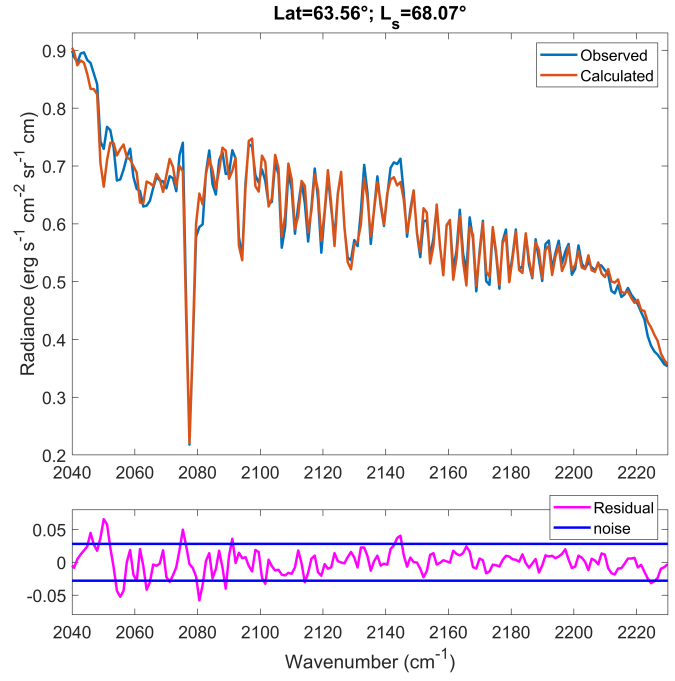

(a) conservative.

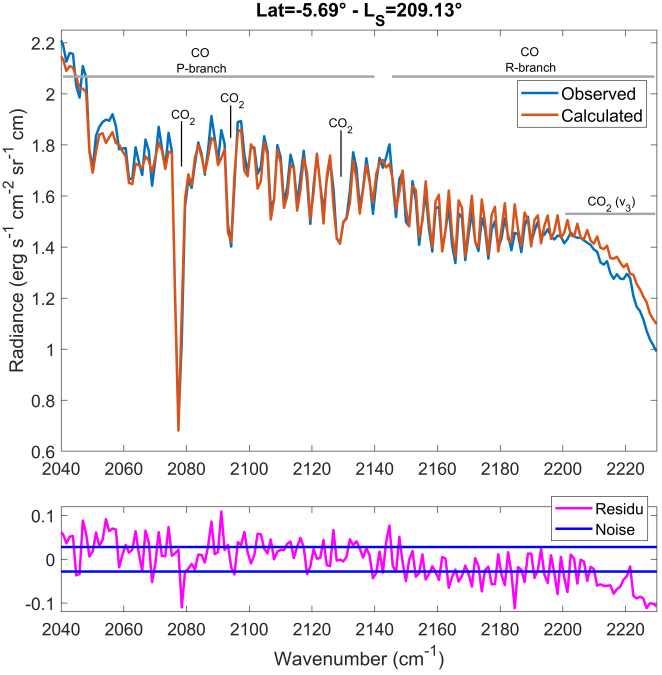

(b)

Figure 4: Example fits among the 16 PFS spectra analysed. For the case (a) with the lowest RMS of the residual (0.019 $\mathrm{erg} \mathrm{s}^{-1} \mathrm{sr}^{-1} \mathrm{~cm}^{-2} \mathrm{~cm}$ ) and, (b) the largest RMS (0.046 $\left.\mathrm{erg} \mathrm{s}^{-1} \mathrm{sr}^{-1} \mathrm{~cm}^{-2} \mathrm{~cm}\right)$.

radiance is significantly higher in this case (around $2 \mathrm{erg} \mathrm{s}^{-1} \mathrm{sr}^{-1} \mathrm{~cm}^{-2} \mathrm{~cm}$ at $2050 \mathrm{~cm}^{-1}$, almost twice larger) than for the lowest RMS case. The increase of the RMS with the radiance level seems to be a general behaviour among the 16 spectra analysed. Overall, we find that the average RMS is very similar to the $\pm \boldsymbol{\sigma}_{\boldsymbol{\epsilon}}$ value considered in $\mathbf{S}_{\boldsymbol{\epsilon}}$, suggesting that this choice for the constrain on the measurement is appropriate and in some cases quite

\section{(0.046 erg s-1 $\left.\mathrm{st}^{-1} \mathrm{~cm}^{-2} \mathrm{~cm}\right)$.}

Figure 5 shows the retrieved CO profiles for three representative cases. Above $5 \mathrm{~km}$ of height, all retrieved profiles present a similar shape as the a priori profile, although not exactly at the same VMR. In the last layer, the maximal difference between the retrieved CO VMRs is equal to $457 \mathrm{ppm}$, with a minimum and a maximum CO VMRs respectively of 514 and $971 \mathrm{ppm}$. Closer to the Martian surface, the variability in the retrieved CO VMRs around the a priori is larger, with a minimum and a maximum of respectively 313 ppm (spectrum recorded in the South Pole during Southern spring) and 1687 ppm (spectrum recorded in mid-latitudes of the Northern hemisphere during Northern autumn), leading to a maximal difference of $1374 \mathrm{ppm}$. 
This departure from the a priori tends to show that the sensitivity of PFS observations is significant close to the surface, and this will be discussed in more details in the next section.

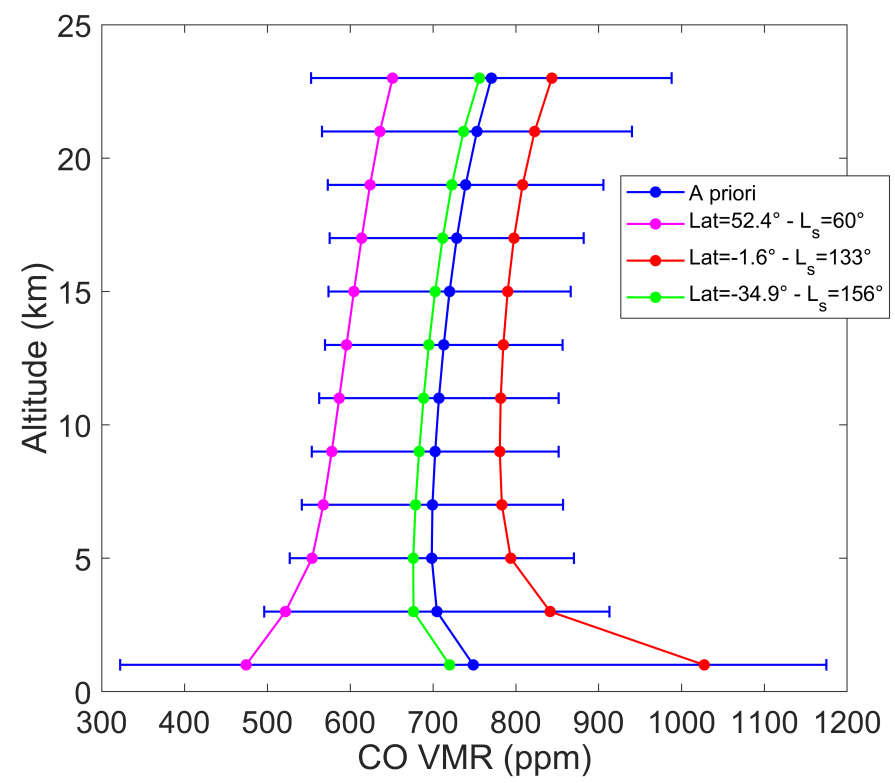

Figure 5: Examples of retrieved CO profiles. The VMRs correspond to the averaged VMRs of each retrieved layer (every $2 \mathrm{~km}$ ). The a priori profile is shown in blue along with the associated a priori variability (square root of the diagonal element of the $\mathbf{S}_{\mathbf{a}}$ ).

The total columns of CO calculated for all spectra vary between $8.83 \times 10^{19}$ molecules $/ \mathrm{cm}^{2}$ and $2.76 \times 10^{20}$ molecules $/ \mathrm{cm}^{2}$. These columns are associated with column-averaged VMRs between $464 \mathrm{ppm}$ and $1115 \mathrm{ppm}$, which are shown in Figure 6 as a function of $\mathrm{L}_{s}$ and latitude. The observed variability is at first sight in agreement with the results of other studies (e.g. [20]). Indeed, previous measurements have reported that CO presents strong latitudinal and seasonal variations, especially in the polar regions, where it is mainly due to variations of the $\mathrm{CO}_{2}$ surface pressure related to its alterning condensation and sublimation. In autumn/winter, $\mathrm{CO}_{2}$ condenses on the polar ice cap, leading to a decrease of the $\mathrm{CO}_{2}$ pressure and, as a result, to an increase of the CO VMR. The reverse happens during spring/summer, when the $\mathrm{CO}_{2}$ ice sublimates. From Figure 6, the column-averaged CO VMRs retrieved in the polar regions during spring/summer are generally lower than those 
retrieved elsewhere, with averaged values around $650 \mathrm{ppm}$ for the North Pole and $500 \mathrm{ppm}$ for the South Pole. Note again that we did not find PFS observations with detectable $\mathrm{CO}$ lines for the poles during the winter. The averaged of the CO VMR calculated for all observations is around $750 \mathrm{ppm}$. These values are in the range of those retrieved from CRISM observations and interestingly capture similar broad patterns of spatial and seasonal variability of CO reported from CRISM [20]. A larger ensemble of PFS observations, recorded at similar locations and times as CRISM measurements, would be required to achieve a better and more insightful comparison between the two satellite datasets.

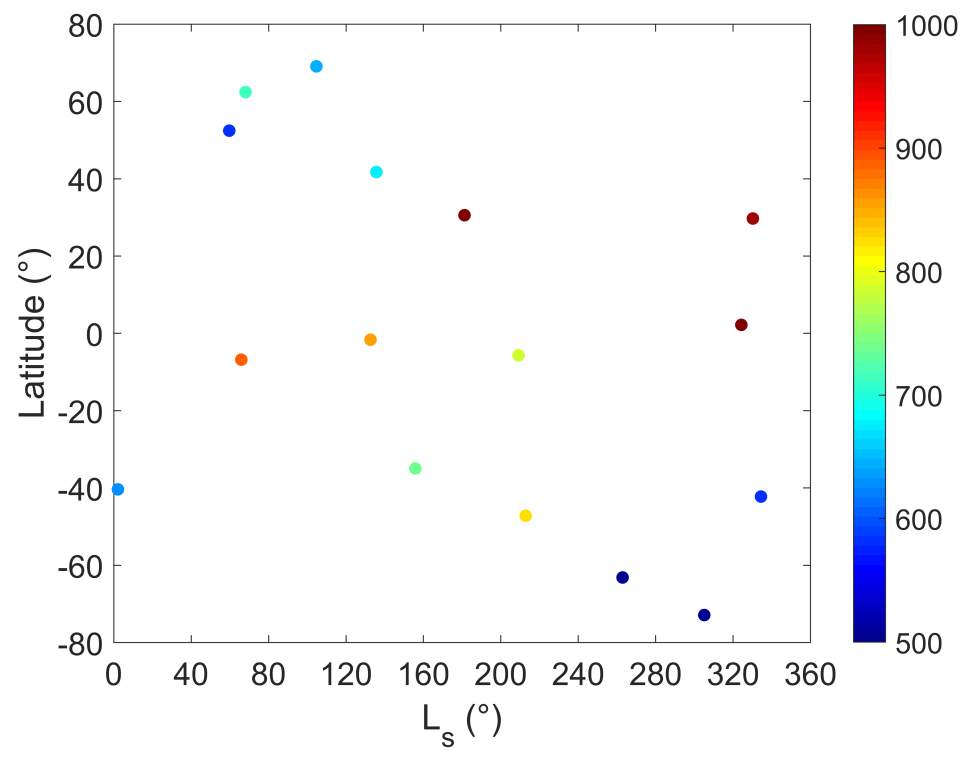

Figure 6: Column-averaged CO VMRs (color scale, ppm) calculated from the retrieved profiles as a function of latitude and $\mathrm{L}_{s}$. Note that the PFS observations correspond to different Martian years.

\subsection{Characterization of the retrieved profiles \\ 6.2.1. Vertical sensitivity}

The vertical sensitivity of the retrieval can be firstly described with the Jacobians, which provide an estimation of the sensitivity of the TOA radiance to a change of the $\mathrm{CO}$ concentration in a certain layer. The Jacobians for one out of the 16 spectra is shown as an example in Figure 7 (note that they are similar for all spectra). As seen from Figure 7, for the entire spectral 
range, the Jacobians reach their largest value close to the surface, showing that this is where the nadir PFS observations have the maximal sensitivity to CO. The values of the Jacobians then decrease with altitude to almost 0 above $15 \mathrm{~km}$, where the sensitivity of PFS measurements to CO is vanishing. An important result that follows is that the sensitivity is altitude dependent and that this a posteriori justifies the idea of fitting a profile rather than a column. It is also worth pointing that the PFS sensitivity to CO is not constant over the whole spectral range used for the retrieval: the instrument is more sensitive to $\mathrm{CO}$ in the strongest R2 to R8 lines $\left(2154-2176 \mathrm{~cm}^{-1}\right)$ for the R-branch and in the P3 to P8 lines $\left(2111-2131 \mathrm{~cm}^{-1}\right)$ for the P-branch.

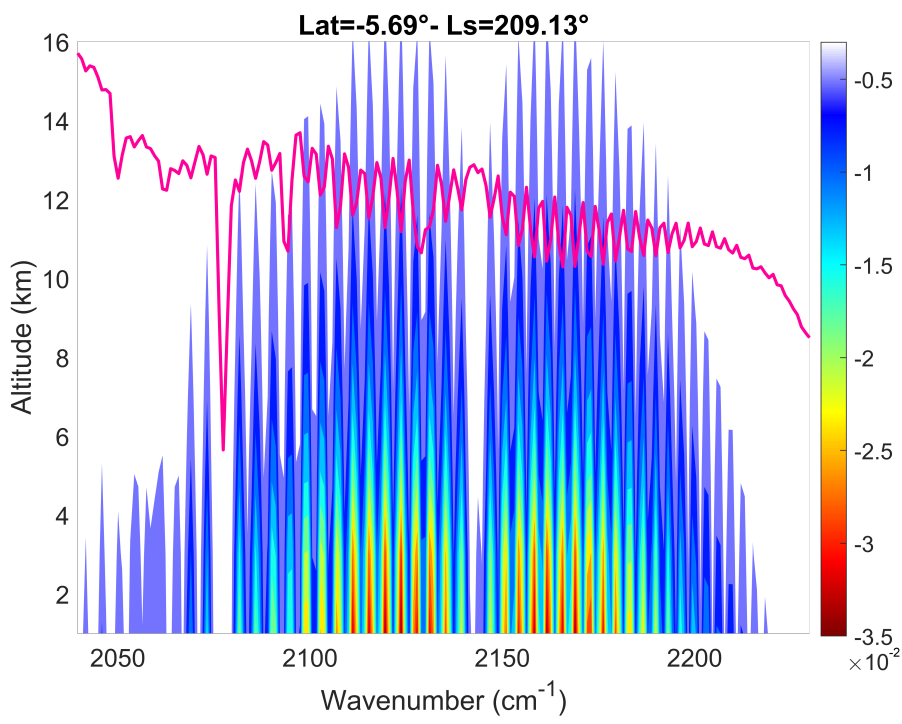

Figure 7: Example of Jacobians (erg s $\mathrm{s}^{-1} \mathrm{~cm}^{-2} \mathrm{sr}^{-1} \mathrm{~cm}$ per scaling factor, color scale) calculated for the PFS observation at $-5.69^{\circ}$ latitude and $132.43^{\circ}$ longitude; $209.13^{\circ} \mathrm{L}_{s}$. The corresponding spectrum is shown in pink.

The maximal sensitivity that PFS has to CO close to the surface is confirmed by the AVK functions, which are shown in Figure 8 for the lowest (a) and highest (b) DOFS cases. For all retrieved layers, the AVK functions peak at the surface, with values ranging between 0.36 and 0.44 . The width of the AVK is large, with each function spanning the range from 0 to about $12 \mathrm{~km}$. This shows that all retrieved layers are correlated with each other and especially that the information in the upper altitudes is mostly driven by the retrieval at the near-surface. While nadir TIR sounders on Earth have 
generally low sensitivity to the surface (e.g. IASI, [26]) this is obviously not the case here. There are two reasons for this: 1) the thermal contrast associated to the 16 selected observations varies between $15 \mathrm{~K}$ and $64 \mathrm{~K}$, which is high compared to the usual values encountered on Earth, and even more importantly 2) the contribution of the reflected solar radiation to the upwelling surface radiance is more significant in the case of Mars than for Earth, which makes the dependency to thermal contrast less relevant. Finally, note that above $15 \mathrm{~km}$, all the AVK functions drop close to 0; the CO retrieval is no longer sensitive to the $\mathrm{CO}$ abundance in these layers.

The averaging kernels show that the retrieved profile is not resolved vertically. In fact among the 16 PFS observations, the DOFS varies between 0.74 and 0.97 . This shows that the only quantity retrieved independently is the total column of CO. The variation of the DOFS is mostly related to that of the signal-to-noise ratio of PFS observations, and hence mainly to surface temperature and reflectivity. The dependence on thermal contrast is second-order and does not affect the values of the DOFS much. There is, however, a positive correlation between the first diagonal element of the AVK matrix (sensitivity of the retrieved CO column in the first layer to the true column in that layer) and the thermal contrast. On the contrary, from the small set of spectra analysed we do not observe a dependence of the vertical sensitivity on the abundance of $\mathrm{CO}$.

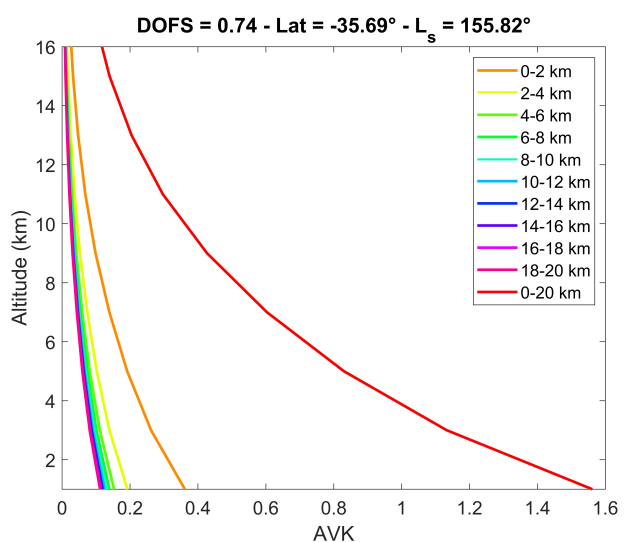

(a)

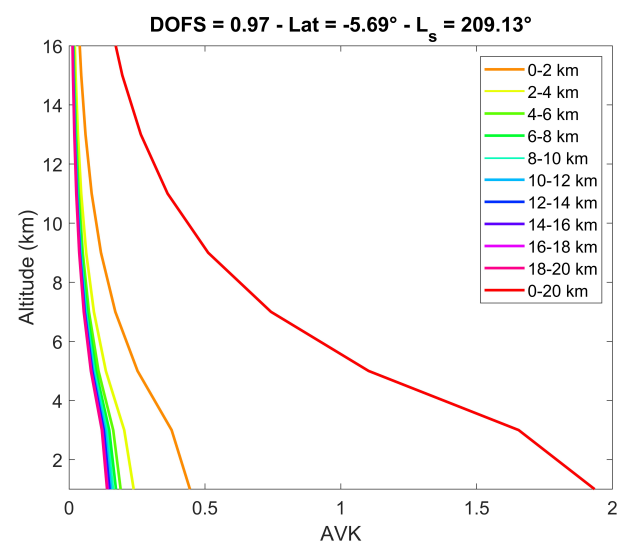

(b)

Figure 8: AVK functions associated to the retrieval of $\mathrm{CO}$ profile for the case with the lowest DOFS (a), and for the case with the highest DOFS (b). The DOFS is indicated on top along with latitude and solar longitude. 


\subsubsection{Error budget}

The different sources of error on the retrieved $\mathrm{CO}$ profile have been evaluated from equations 8, 9, 10 and 11 along with the total error. Figure 9 shows the vertical distribution of these errors for two cases: the one with the lowest uncertainty (Figure 9.a) and the one with the highest uncertainty (Figure 9.b). For all 16 spectra, the largest error is found at the surface (where the prior uncertainty is the largest), and varies between $26 \%$ and $32 \%$. The retrieval error then decreases with height. Above $5 \mathrm{~km}$, it stays almost constant and varies between $10 \%$ and $13 \%$. Overall, at all altitudes, the prior uncertainty is reduced by around $50 \%$, showing the improvement in the knowledge of the $\mathrm{CO}$ vertical distribution brought by PFS measurements. The total retrieval error on the total column of $\mathrm{CO}$ is between 5 and $15 \%$, which is promising in the perspective to study the CO spatial and temporal variability on Mars.

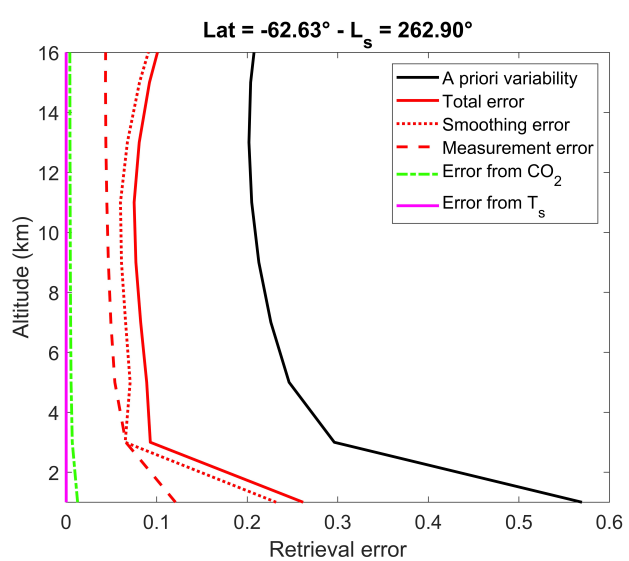

(a)

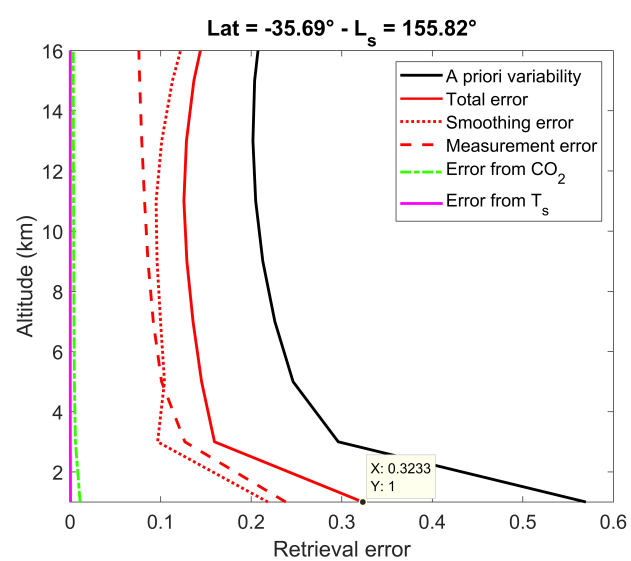

(b)

Figure 9: Vertical distribution of the total error on the retrieved CO profile and of the different sources of uncertainty. Two cases are shown: the one with the lowest total error (a) and the one with the highest error (b). The a priori variability is shown as the black line.

As seen from Figure 9, the two main sources of uncertainty are the smoothing and the measurement errors, which have very similar contributions to the total retrieval error. Above $6 \mathrm{~km}$, the smoothing error is generally the largest and varies between $6 \%$ and $11 \%$. The measurement error is lower in this altitude range and varies between 5 and $9 \%$. At the surface, the 


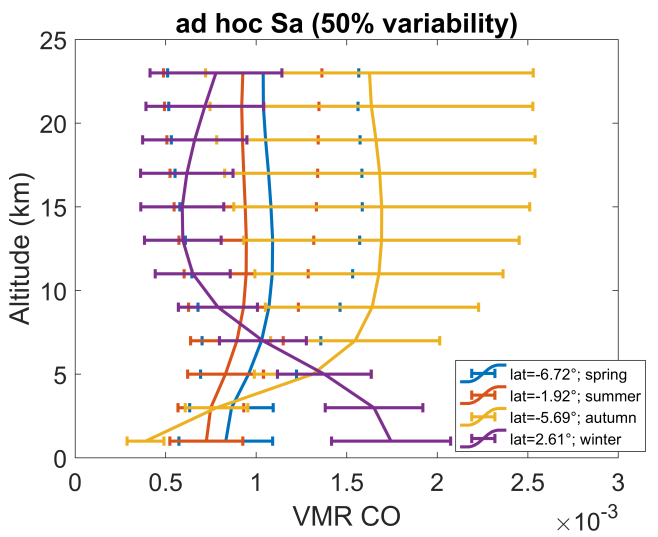

(a)

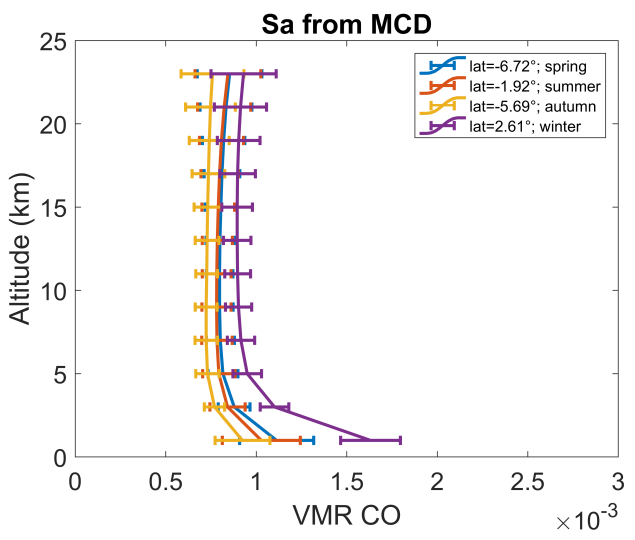

(b)

Figure 10: The retrieved CO profiles on the equator for different season by testing two $a$ priori covariance matrices. 
similar fits quality of PFS observations. The baseline chosen for this work is the one recommended by the PI team of PFS (hereafter called "PILS") and in this section, we evaluate the impact of this choice on the retrieved $\mathrm{CO}$ profile by also evaluating the results obtained with the modified Hamming function (HG). This is done by calculating, for each observation, the relative difference (RD) between the retrieved CO profiles using the two ILS models as $R D=\frac{\left(C O_{P I L S}-C O_{H G}\right) \times 100}{C O_{P I L S}}$. The average relative difference for all the retrieved layers is shown in Figure 11. It is clear from this figure that the choice of the ILS function has a small but non negligible effect on the retrieved CO profile. The CO VMR is systematically higher when using the PILS, with a maximum relative difference at the surface, where it reaches $9 \%$. It then decreases to around 3\% at higher altitudes. On the total column of CO, this translates to relative differences around $5 \%$ on average. It is useful to have these differences in mind for future work, as these can be taken as a systematic uncertainty on the retrieved CO profile and columns.

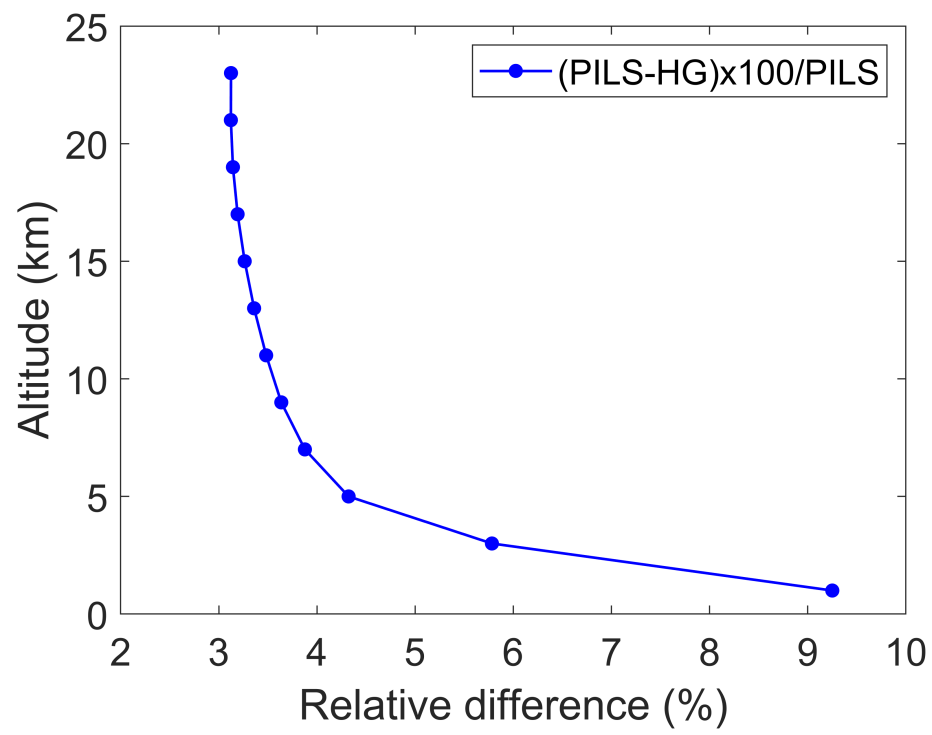

Figure 11: Averaged relative difference between the CO profiles retrieved with the ILS recommended by the PI team of PFS and the HG ILS adjusted in this work. 


\section{Conclusions}

A first attempt to retrieve $\mathrm{CO}$ vertical profiles from nadir observations on Mars has been performed for the first time. This has been achieved on high spectral resolution measurements made by the PFS instrument on Mars Express, in the spectral region of the 1-0 fundamental band of $\mathrm{CO}$ (centered at $2143 \mathrm{~cm}^{-1}$ ). Despite the fact that only a very small set of spectra with sufficient signal-to-noise ratios has been considered in this exploratory study, our results have clearly demonstrated the potential of using this spectral range for monitoring $\mathrm{CO}$ spatially and temporally on single PFS observations, except in the polar winters. The CO profiles have been retrieved using the Optimal Estimation Method, with a priori information (a priori profile and variance-covariance matrix) built from a large ensemble of global model profiles, which we have shown to provide appropriate constraints. The CO profiles retrieved are very weakly resolved vertically, with the PFS spectra carrying no more than one piece of information. The vertical sensitivity is, however, altitude-dependent (sensitivity from 0 to about $20 \mathrm{~km}$, maximal at the surface) and this alone justifies the retrieval of the profile. The errors on each level of the profile vary between $25 \%$ close to the surface to less than $10 \%$ above $3 \mathrm{~km}$, due to the combined contribution of the measurement noise and the smoothing error. This translates to an error on the retrieved column or the column-averaged VMR of 5 to $15 \%$ depending on the scene. Other sources of errors have been identified and we have characterized in particular the one introduced by the uncertainty on the PFS ILS. In all cases we found that the PFS measurements substantially reduce the prior uncertainty on the $\mathrm{CO}$ profile and the column, and, as a result, that the retrieved $\mathrm{CO}$ abundances allow capturing the spatial and seasonal variability of $\mathrm{CO}$ on Mars. We found a preliminary agreement, considering the different kind of datasets that are compared, with CO abundances retrieved from the CRISM spectrometer [20]. Generally, the possibility of retrieving CO with good accuracy from PFS single observations in the 1-0 band is very promising and stimulates larger-scale retrievals, which would provide strong constraints on the cycle of carbon species in the lowest (below $15 \mathrm{~km}$ ) Martian atmosphere.

\section{Acknowledgements}

This work is funded by the FNRS CRAMIC project under grant number T.0171.16. PFS is funded by the Italian Space Agency under grant 20182-HH.0. Sophie Bauduin is Chargée de Recherches with FRS-FNRS. The 
authors would like to thank the LMD for providing the access to the Mars Climate Database, needed to build the a priori vertical profile CO and the associated covariance matrix.

\section{References}

[1] L. D. Kaplan, J. Connes, and P. Connes, "Carbon monoxide in the Martian atmosphere," The Astrophysical Journal, vol. 157, pp. L187L192, 1969.

[2] M. B. McElroy and T. M. Donahue, "Stability of the Martian Atmosphere," Science, vol. 177, pp. 986-988, Sept. 1972.

[3] T. D. Parkinson and D. M. Hunten, "Spectroscopy and Acronomy of $\mathrm{O}_{2}$ on Mars.," Journal of Atmospheric Sciences, vol. 29, pp. 1380-1390, Oct. 1972.

[4] S. K. Atreya and Z. G. Gu, "Stability of the Martian atmosphere: Is heterogeneous catalysis essential?," Journal of Geophysical Research, vol. 99, pp. 13133-13145, June 1994.

[5] S. K. Atreya and Z. G. Gu, "Photochemistry and stability of the atmosphere of Mars," Advances in Space Research, vol. 16, Aug. 1995.

[6] H. Nair, M. Allen, A. D. Anbar, Y. L. Yung, and R. T. Clancy, "A photochemical model of the martian atmosphere," Icarus, vol. 111, pp. 124150, Sept. 1994.

[7] F. Forget, F. Hourdin, R. Fournier, C. Hourdin, O. Talagrand, M. Collins, S. R. Lewis, P. L. Read, and J.-P. Huot, "Improved general circulation models of the Martian atmosphere from the surface to above 80 km," Journal of Geophysical Research: Planets, vol. 104, no. E10, pp. 24155-24175, 1999.

[8] L. Neary and F. Daerden, "The GEM-Mars general circulation model for Mars: Description and evaluation," Icarus, vol. 300, pp. 458-476, 2018.

[9] S. K. Atreya and J. E. Blamont, "Stability of the Martian atmosphere Possible role of heterogeneous chemistry," Geophysical Research Letters, vol. 17, pp. 287-290, Mar. 1990. 
[10] F. Lefèvre, J.-L. Bertaux, R. T. Clancy, T. Encrenaz, K. Fast, F. Forget, S. Lebonnois, F. Montmessin, and S. Perrier, "Heterogeneous chemistry in the atmosphere of Mars," Nature, vol. 454, pp. 971-975, Aug. 2008.

[11] V. Formisano, S. Atreya, T. Encrenaz, N. Ignatiev, and M. Giuranna, "Detection of Methane in the Atmosphere of Mars," Science, vol. 306, pp. 1758-1761, Dec. 2004.

[12] K. Zahnle, R. S. Freedman, and D. C. Catling, "Is there methane on Mars?," Icarus, vol. 212, pp. 493-503, Apr. 2011.

[13] C. R. Webster, P. R. Mahaffy, S. K. Atreya, J. E. Moores, G. J. Flesch, C. Malespin, C. P. McKay, G. Martinez, C. L. Smith, J. Martin-Torres, J. Gomez-Elvira, M.-P. Zorzano, M. H. Wong, M. G. Trainer, A. Steele, D. Archer, B. Sutter, P. J. Coll, C. Freissinet, P.-Y. Meslin, R. V. Gough, C. H. House, A. Pavlov, J. L. Eigenbrode, D. P. Glavin, J. C. Pearson, D. Keymeulen, L. E. Christensen, S. P. Schwenzer, R. NavarroGonzalez, J. Pla-García, S. C. R. Rafkin, Á. Vicente-Retortillo, H. Kahanpää, D. Viudez-Moreiras, M. D. Smith, A.-M. Harri, M. Genzer, D. M. Hassler, M. Lemmon, J. Crisp, S. P. Sander, R. W. Zurek, and A. R. Vasavada, "Background levels of methane in Mars' atmosphere show strong seasonal variations," Science, vol. 360, pp. 1093-1096, June 2018.

[14] V. A. Krasnopolsky, "Spectroscopic mapping of Mars CO mixing ratio: Detection of north-south asymmetry," Journal of Geophysical Research (Planets), vol. 108, p. 5010, Feb. 2003.

[15] T. Encrenaz, T. Fouchet, R. Melchiorri, P. Drossart, B. Gondet, Y. Langevin, J.-P. Bibring, F. Forget, and B. Bézard, "Seasonal variations of the martian CO over Hellas as observed by OMEGA/Mars Express," Astronomy \& Astrophysics, vol. 459, pp. 265-270, Nov. 2006.

[16] F. Billebaud, J. Brillet, E. Lellouch, T. Fouchet, T. Encrenaz, V. Cottini, N. Ignatiev, V. Formisano, M. Giuranna, A. Maturilli, and F. Forget, "Observations of CO in the atmosphere of Mars with PFS onboard Mars Express," Planetary and Space Science, vol. 57, pp. 1446-1457, Oct. 2009. 
[17] G. Sindoni, V. Formisano, and A. Geminale, "Observations of water vapour and carbon monoxide in the Martian atmosphere with the SWC of PFS/MEX," Planetary and Space Science, vol. 59, no. 2-3, pp. 149$162,2011$.

[18] M. Giuranna, P. Wolkenberg, D. Grassi, A. Aronica, S. Aoki, D. Scaccabarozzi, B. Saggin, V. Formisano, and the PFS team, "New dataset of atmospheric parameters retrieved by PFS-MEx." Abstract Scientific Workshop: From Mars Express to ExoMars, 2018.

[19] M. D. Smith, M. J. Wolff, R. T. Clancy, and S. L. Murchie, "Compact Reconnaissance Imaging Spectrometer observations of water vapor and carbon monoxide," Journal of Geophysical Research: Planets, vol. 114, no. E2, 2009.

[20] M. D. Smith, F. Daerden, L. Neary, and A. Khayat, "The climatology of carbon monoxide and water vapor on Mars as observed by CRISM and modeled by the GEM-Mars general circulation model," Icarus, vol. 301, pp. 117-131, 2018.

[21] R. T. Clancy, D. O. Muhleman, and B. M. Jakosky, "Variability of carbon monoxide in the Mars atmosphere," Icarus, vol. 55, no. 2, pp. 282301, 1983.

[22] E. Lellouch, J. J. Goldstein, S. W. Bougher, G. Paubert, and J. Rosenqvist, "First absolute wind measurements in the middle atmosphere of Mars," The Astrophysical Journal, vol. 383, pp. 401-406, 1991.

[23] A. S. Khayat, G. L. Villanueva, M. J. Mumma, and A. T. Tokunaga, "A search for $\mathrm{SO}_{2}, \mathrm{H}_{2} \mathrm{~S}$ and $\mathrm{SO}$ above Tharsis and Syrtis volcanic districts on Mars using ground-based high-resolution submillimeter spectroscopy," Icarus, vol. 253, pp. 130-141, 2015.

[24] A. S. J. Khayat, G. L. Villanueva, M. J. Mumma, and A. T. Tokunaga, "A deep search for the release of volcanic gases on Mars using groundbased high-resolution infrared and submillimeter spectroscopy: sensitive upper limits for OCS and $\mathrm{SO}_{2}, "$ Icarus, vol. 296, pp. 1-14, 2017.

[25] S. Robert, C. Camy-Peyret, F. Daerden, M. De Mazière, E. De Wachter, L. Neary, S. Vandenbussche, and A. Vandaele, "Two test-cases for synergistic detections in the Martian atmosphere: Carbon monoxide and 
methane," Journal of Quantitative Spectroscopy and Radiative Transfer, vol. 189, pp. 86-104, 2017.

[26] S. Bauduin, L. Clarisse, M. Theunissen, M. George, D. Hurtmans, C. Clerbaux, and P.-F. Coheur, "IASI's sensitivity to near-surface carbon monoxide (CO): Theoretical analyses and retrievals on test cases," Journal of Quantitative Spectroscopy and Radiative Transfer, vol. 189, pp. 428-440, 2017.

[27] C. D. Rodgers, Inverse methods for atmospheric sounding: theory and practice, vol. 2. World scientific, 2000.

[28] V. Formisano, F. Angrilli, G. Arnold, S. Atreya, G. Bianchini, D. Biondi, A. Blanco, M. Blecka, A. Coradini, L. Colangeli, et al., "The planetary Fourier spectrometer (PFS) onboard the European Mars Express mission," Planetary and Space Science, vol. 53, no. 10, pp. 963-974, 2005.

[29] M. Giuranna, V. Formisano, D. Biondi, A. Ekonomov, S. Fonti, D. Grassi, H. Hirsch, I. Khatuntsev, N. Ignatiev, M. Michalska, et al., "Calibration of the Planetary Fourier Spectrometer short wavelength channel," Planetary and Space Science, vol. 53, no. 10, pp. 975-991, 2005 .

[30] M. Giuranna, V. Formisano, D. Biondi, A. Ekonomov, S. Fonti, D. Grassi, H. Hirsch, I. Khatuntsev, N. Ignatiev, M. Malgoska, et al., "Calibration of the Planetary Fourier Spectrometer long wavelength channel," Planetary and Space Science, vol. 53, no. 10, pp. 993-1007, 2005.

[31] B. Barret, D. Hurtmans, M. R. Carleer, M. De Mazière, E. Mahieu, and P.-F. Coheur, "Line narrowing effect on the retrieval of $\mathrm{HF}$ and $\mathrm{HCl}$ vertical profiles from ground-based FTIR measurements," Journal of Quantitative Spectroscopy and Radiative Transfer, vol. 95, no. 4, pp. 499-519, 2005.

[32] P.-F. Coheur, B. Barret, S. Turquety, D. Hurtmans, J. Hadji-Lazaro, and C. Clerbaux, "Retrieval and characterization of ozone vertical profiles from a thermal infrared nadir sounder," Journal of Geophysical Research: Atmospheres, vol. 110, no. D24, 2005. 
[33] C. Clerbaux, P.-F. Coheur, D. Hurtmans, B. Barret, M. Carleer, R. Colin, K. Semeniuk, J. McConnell, C. Boone, and P. Bernath, "Carbon monoxide distribution from the ACE-FTS solar occultation measurements," Geophysical research letters, vol. 32, no. 16, 2005.

[34] C. D. Rodgers and B. J. Connor, "Intercomparison of remote sounding instruments," Journal of Geophysical Research (Atmospheres), vol. 108, p. 4116, Feb. 2003.

[35] P. Wolkenberg, M. Giuranna, D. Grassi, A. Aronica, S. Aoki, D. Scaccabarozzi, and B. Saggin, "Characterization of dust activity on Mars from MY27 to MY32 by PFS-MEX observations," Icarus, vol. 310, pp. 32-47, 2018.

[36] M. Giuranna and et al., "The current weather and climate of Mars: 12 years of atmospheric monitoring by the Planetary Fourier Spectrometer on Mars Express." Icarus, 2019 (submitted).

[37] D. Grassi, N. I. Ignatiev, L. V. Zasova, A. Maturilli, V. Formisano, G. A. Bianchini, and M. Giuranna, "Methods for the analysis of data from the Planetary Fourier Spectrometer on the Mars Express Mission," Planetary and Space Science, vol. 53, pp. 1017-1034, Aug. 2005.

[38] F. Poulet, C. Quantin-Nataf, H. Ballans, K. Dassas, J. Audouard, J. Carter, B. Gondet, L. Lozach, J.-C. Malapert, C. Marmo, et al., "PSUP: A Planetary SUrface Portal," Planetary and Space Science, 2017.

[39] C. Quantin-Nataf, L. Lozac'h, P. Thollot, D. Loizeau, B. Bultel, J. Fernando, P. Allemand, F. Dubuffet, F. Poulet, A. Ody, et al., "MarsSI: Martian surface data processing information system," Planetary and Space Science, vol. 150, pp. 157-170, 2018.

[40] J. Audouard, F. Poulet, M. Vincendon, J.-P. Bibring, F. Forget, Y. Langevin, and B. Gondet, "Mars surface thermal inertia and heterogeneities from OMEGA/MEX," Icarus, vol. 233, pp. 194-213, 2014.

[41] I. E. Gordon, L. S. Rothman, C. Hill, R. V. Kochanov, Y. Tan, P. F. Bernath, M. Birk, V. Boudon, A. Campargue, K. V. Chance, B. J. Drouin, J.-M. Flaud, R. R. Gamache, J. T. Hodges, D. Jacquemart, 
V. I. Perevalov, A. Perrin, K. P. Shine, M.-A. H. Smith, J. Tennyson, G. C. Toon, H. Tran, V. G. Tyuterev, A. Barbe, A. G. Császár, V. M. Devi, T. Furtenbacher, J. J. Harrison, J.-M. Hartmann, A. Jolly, T. J. Johnson, T. Karman, I. Kleiner, A. A. Kyuberis, J. Loos, O. M. Lyulin, S. T. Massie, S. N. Mikhailenko, N. Moazzen-Ahmadi, H. S. P. Müller, O. V. Naumenko, A. V. Nikitin, O. L. Polyansky, M. Rey, M. Rotger, S. W. Sharpe, K. Sung, E. Starikova, S. A. Tashkun, J. V. Auwera, G. Wagner, J. Wilzewski, P. Wcisło, S. Yu, and E. J. Zak, "The HITRAN2016 molecular spectroscopic database," Journal of Quantitative Spectroscopy and Radiative Transfer, vol. 203, pp. 3-69, 2017.

[42] G. Li, I. E. Gordon, L. S. Rothman, Y. Tan, S.-M. Hu, S. Kassi, A. Campargue, and E. S. Medvedev, "Rovibrational Line Lists for Nine Isotopologues of the CO Molecule in the $\mathrm{X}^{1} \Sigma^{+}$Ground Electronic State," The Astrophysical Journal Supplement Series, vol. 216, p. 15, Jan. 2015.

[43] M. Giuranna, S. Viscardy, F. Daerden, L. Neary, G. Etiope, D. Oehler, V. Formisano, A. Aronica, P. Wolkenberg, S. Aoki, A. Cardesn-Moinelo, J. Marn-Yaseli de la Parra, D. Merritt, and M. Amoroso, "Independent confirmation of a methane spike on Mars and a source region east of Gale Crater," Nature Geoscience, 2019.

[44] J. Kauppinen and P. Saarinen, "Line-shape distortions in misaligned cube corner interferometers," Applied Optics, vol. 31, pp. 69-74, 1992.

[45] E. Millour, F. Forget, A. Spiga, M. Vals, V. Zakharov, L. Montabone, F. Lefèvre, F. Montmessin, J.-Y. Chaufray, M. A. Lòpez-Valverde, F. González-Galindo, S. R. Lewis, P. L. Read, M. C. Desjean, F. Cipriani, and the MCD development team, "The Mars climate database (version 5.3)," in Scientific Workshop: From Mars Express to ExoMars (ESA-ESAC), 2018. 